\title{
Link between low-frequency noise enhancement and high-frequency oscillations in GaN-based planar nanodiodes: a Monte Carlo study
}

\author{
A. Íñiguez-de-la-Torre, I. Íñiguez-de-la-Torre, J. Mateos and T. González \\ Departamento de Física Aplicada, Universidad de Salamanca, Plaza de la Merced s/n, \\ 37008 Salamanca, Spain
}

\begin{abstract}
We present a spectral analysis of time sequences of current, calculated by means of Monte Carlo simulations, in GaN-based asymmetric nanodiodes, devices that are potential candidates to exhibit Gunn oscillations. It is found that the low-frequency noise increases significantly for biases close to the threshold voltage of Gunn oscillations, taking place at much higher frequencies of hundreds of GHz. Due to the inherent difficulty in detecting so fast fluctuations, the measurement of the lowfrequency noise can be a quite useful tool for predicting current oscillations at sub-THz frequencies in these devices.
\end{abstract}


Gunn-phenomena based devices have been investigated as a source of microwave and millimetre wave radiation since almost 50 years. Most of the research works have been directed to improve power, high frequency and heat dissipation of Gunn oscillators, which in the last years reveal GaN as a promising material in this field. The search for materials with high electrical strength to allow high power generation points to $\mathrm{GaN}$ as compared to GaAs because of its higher energy gap. The second point is the high frequency issue, in which the higher peak and saturation velocity, and lower energy relaxation time of $\mathrm{GaN}$ provide also better performances [2]. Finally, heat dissipation has been realized to be a big problem for fabricating GaN Gunn oscillators. Mainly vertical GaN structures were explored so far, with little success [5]. Planar topologies as those proposed in ref. [6] allowing for more efficient heat dissipation can be a good solution. The approach we follow in this work for the case of GaN is the use of parallel arrays of planar asymmetric nanodiodes. The device, so-called selfswitching diode (SSD), proposed by A. M. Song e. al. in 2003 [8] was intended for logic circuit design. Soon after, SSDs demonstrated detection capability up to $110 \mathrm{GHz}$ at room temperature [9] and up to $2.5 \mathrm{THz}$ at $10 \mathrm{~K}$ [10]. Monte Carlo (MC) simulations have been used to study the SSD static, dynamic and noise properties (see $[13,15]$ and references therein). Recently, the functionality of this seemingly simple device has reached one more step: the ability of power generation based on Gunn oscillations [16]. Our research goes towards a compact, cheap and room temperature source above $1 \mathrm{THz}$, using GaN-based SSDs. Experimental results of Gunn oscillations in these devices have not been reported yet but numerical MC simulations predict high frequency oscillations [17].

Up to now it is still quite tricky to carry out experimental measurements at hundreds of GHz. For that reason, alternative methods of analysis become crucial into the field of $\mathrm{THz}$ or sub-THz electronic devices, in particular to detect the presence of oscillations in this frequency range. A first possible indicator is related to the kinks that sometimes appear in the $I-V$ curves for applied voltages at which the onset of instabilities take place $[\mathrm{xx}]$, indicating the transition from a passive- to a generation-state. In the case of the MC simulations of GaN-based SSDs, below presented in detail, no kink is exhibited by the $I-V$ curve even though clear Gunn oscillations in the current sequences are observed. Another prediction method already suggested by Starikov et al. [19] consists in the use of the low-frequency noise in the current $S_{I}(0)$ (in the plateau beyond $1 / \mathrm{f}$ and 
g-r noise) as indicator of the onset of oscillations since it exhibits a significant enhancement for voltages approaching the threshold of the oscillations.

The aim of this work is to check, by means of MC simulations, if this behaviour takes place in the GaN SSDs under study, since they are promising devices for room temperature THz emission by exploiting Gunn Effect. Thus, we analyze the possibility of indirectly detecting the presence of current oscillations by the enhancement of lowfrequency noise.

The onset of current oscillations in GaN-based SSDs has been analyzed by means of an ensemble MC simulator self-consistently coupled with a 2D Poisson solver. The conduction band of $\mathrm{GaN}$ is modelled by three non-parabolic spherical valleys $\left(\Gamma_{1}, \mathrm{U}\right.$ and $\Gamma_{3}$ ), and the considered scattering mechanisms are acoustic, optical and intervalley phonons, ionized impurities and piezoelectric scattering. Since the simulation tool (code developed by our group) is a 2D solver, just "top-view" simulations of the channel have been performed [22]. In order to account for the 3D real topology we include the fixed charges of the whole layer structure as a "virtual" net doping $N_{D b}$ to the channel, considered when solving Poisson Equation but ignored as a source of scattering. We also include a negative surface charge density $\sigma$ at the semiconductor-air interfaces. More information about the simulation model can be found in [22].

To perform a frequency analysis of electrical fluctuations and to detect the presence of current oscillations, the time-domain current sequences obtained from the MC simulations are Fourier transformed into the frequency domain to determine the current noise spectral density $S_{I}(f)$. The zero frequency value $S_{I}(0)$, corresponding to the noise measured in experiments in the plateau beyond $1 / \mathrm{f}$ and g-r noise, will be used as the indicator for the occurrence of oscillations. Both $S_{I}$ and current values we present in the result section are normalized in such a way that just multiplying them by the value of the sheet electron density in the channel we will obtain $\mathrm{A}^{2} \mathrm{~s}$ and A respectively.

The typical top-view geometry of the SSD channel is shown in Fig. 1(b), where the dimensions used in our simulations are indicated. Two favourable conditions for the onset of Gunn oscillations take place in this structure: i) the electric field is well focused at the cathode side of the channel because of the presence of the vertical trenches, and ii) the electron concentration is increased by the side field effect when biasing the anode with a positive voltage. 
In the next section we present the results for three SSDs with the same value of the virtual doping, $N_{D b}=2 \times 10^{17} \mathrm{~cm}^{-3}$, and different values of the surface charge density, since this parameter is decisive for achieving current oscillations. A large value of $\sigma$ contributes to deplete the channel and higher voltages are necessary for obtaining oscillations. In the first diode we have set $\sigma / q=-0.25 \times 10^{12} \mathrm{~cm}^{-2}$, in the second one $\sigma / q=-1.0 \times 10^{12} \mathrm{~cm}^{-2}$ and $\sigma / q=-1.5 \times 10^{12} \mathrm{~cm}^{-2}$ in the last one.

Figure 2 shows the low-frequency current noise as a function of the applied voltage for the first diode, the one with the lower value considered for the surface charge density, i.e. $\sigma / q=-0.25 \times 10^{12} \mathrm{~cm}^{-2}$. Current sequences and noise spectra are also plotted as insets in order to get more information about what happens in the diode for several voltages at which $S_{I}(0)$ exhibits significant variations while in the $I-V$ curves, shown in Fig. 3(a), there is no kink. As can be observed, the threshold for the onset of Gunn oscillations is about $36 \mathrm{~V}$. Below this voltage, starting from the Nyquist value at equilibrium, the noise exhibits a slight increase with the bias due to electron heating. For voltages higher than $36 \mathrm{~V}$, a remarkable enhancement of the low-frequency noise is detected, in parallel to the transition from a passive- to a generation-state as evidenced by the current sequence and its corresponding spectral density at $45 \mathrm{~V}$. Once the oscillations are mature (voltages above $45 \mathrm{~V}), S_{I}(0)$ decreases and then, at around $53 \mathrm{~V}$, it increases again because of the onset of further frequency components, as observed for $60 \mathrm{~V}$. For higher voltages we find once more the previously explained behaviour, i.e. a drop in the noise up to $75 \mathrm{~V}$ due to the presence of clearly established oscillations followed by another rise according to the incorporation of more harmonics, as occurs at $100 \mathrm{~V}$.

In order to confirm that the enhancement of the low-frequency noise is certainly due to the onset of current oscillations and further spectral components, simulation results of two more SSDs are presented in Fig. 3. The sole variation with respect to SSD1 made in these diodes, called SSD2 and SSD3, is just considering $\sigma / q=-1.0 \times 10^{12} \mathrm{~cm}^{-2}$ and $\sigma / q=-1.5 \times 10^{12} \mathrm{~cm}^{-2}$, respectively. A higher value of $\sigma$ leads to a stronger channel depletion that makes necessary higher voltages to achieve, first, current conduction through the diode, and then Gunn oscillations, as shown in Fig. 3(a). In SSD2 oscillations are more difficult to be obtained, being necessary the application of a voltage around $80 \mathrm{~V}$, while for SSD3 the effective width of the channel is not enough 
to achieve current oscillations even at $100 \mathrm{~V}$. In fact, the current level is much lower than in the other diodes.

With regard to the low-frequency noise [see Fig. 3(b)], the curve corresponding to SSD2 exhibits a more or less constant value up to $80 \mathrm{~V}$ (with a slight increase due to electron heating) and then rises considerably, coinciding with the onset of oscillations at that voltage. For a lower value of $\sigma$, which is the case of SSD1 presented before, such a bump takes place at lower bias, at $36 \mathrm{~V}$, while for a higher value of $\sigma$, as occurs for SSD3, $S_{I}(0)$ remains practically constant since no oscillation is achieved.

In this work we propose to make use of the low-frequency noise spectral density in order to infer if Gunn oscillations take place in GaN SSDs. MC results exhibit a clear enhancement of the low-frequency noise just for voltages corresponding to the onset of current oscillations and also when new harmonic components emerge. Thus, the measurement of $S_{I}(0)$ could be useful to identify the presence of oscillations in structures where they take place at very high (sub-THz) frequencies, like the GaN SSDs analyzed here, and thus circumvent the well known difficulties of experimental measurements in this frequency range.

This work has been partially supported by the EC through the ROOTHz Project ICT2009-243845, by the Dirección General de Investigación (MICINN) and FEDER through Project TEC2010-15413 and by the Junta de Castilla y León through Project GR270. 
[1] J. B. Gunn, Solid State Commun., 1, 88 (1963)

[2] E. Alekseev and D. Pavlidis, Solid-State Electronics 44, 941-947 (2000).

[3] K. Mutambaa, O. Yilmazoglua, C. Sydloa, M. Mira, S. Hubbardb, G. Zhaob, I. Daumillerc and D. Pavlidis, Superlatt. Microstruct., vol. 40, p. 363, 2006.

[4] O. Yilmazoglu, K. Mutamba, D. Pavlidis and T. Karaduman, Electronics Lett., vol. 43, p. 480, 2007.

[5] O. Yilmazoglu, K. Mutamba, D. Pavlidis and T. Karaduman, IEEE Trans. Electron Dev., vol. 55, p. 1563, 2008.

[6] N. J. Pilgrim, A. Khalid, G. M. Dunn, and D. R. S. Cumming, Semicond. Sci. Technol. 23, 075013 (2008).

[7] Ch. Li, A. Khalid, N. J. Pilgrim, M. C. Holland, G. M. Dunn, and D. R. S. Cumming, 35th International Conference on Infrared Millimeter and Terahertz Waves, 2010. DOI: 10.1109/ICIMW.2010.5612507

[8] A. M. Song, M. Missous, P. Omling, A. R. Peaker, L. Samuelson and W. Seifert, Appl. Phys. Lett. 83, 1881 (2003).

[9] C. Balocco, A. M. Song, M. Aberg, A. Forchel, T. González, J. Mateos, I. Maximov, M. Missous, A. A. Rezazadeh, J. Saijets, L. Samuelson, D. Wallin, K. Williams, L. Worschech and H. Q. Xu, Nano Letters 5, 1423 (2005).

[10] C. Balocco, M. Halsall, N. Q. Vinh and A. M. Song, J. Phys.: Condens. Matter 20, 384203 (2008).

[11] Mateos J., Vasallo B. G., Pardo D. and González T., Appl. Phys. Lett. 86, 212103 (2005).

[12] K. Y. Xu, X. F. Lu, A. M. Song and G. Wang, Appl. Phys. Lett. 92, 163503 (2008).

[13] I. Iñiguez-de-la-Torre, J. Mateos, D. Pardo, A. M. Song and T. González, Appl. Phys. Lett. 94, 093512 (2009).

[14] I. Iñiguez-de-la-Torre, J. Mateos, D. Pardo and González T., J. Appl. Phys. 103, 024502 (2008). 
[15] K. Y. Xu, X. F. Lu, A. M. Song and G. Wang, J. Appl. Phys. 103, 113708 (2008).

[16] K. Y. Xu, G. Wang and A. M. Song, Appl. Phys. Lett. 93, 233506 (2008).

[17] T. González, I. Iñiguez-de-la Torre, D. Pardo, J. Mateos and A. M. Song, Journal of Physics: Conference Series 193, 012018 [1-4] (2009).

[18] T. González, I. Íñiguez-de-la-Torre, D. Pardo, A. M. Song and J. Mateos, 22th International Conference on Indium Phosphide and Related Materials (IPRM 2010), Takamatsu, Japan, May 31-June 4, (2011).

[19] E. Starikov, P. Shiktorov, V. Gružinskis, L. Reggiani, L. Varani and J.C. Vaissière, AIP Conf. Proc. 780, 791 (2005).

[20] P. Shiktorov, E. Starikov, V. Gružinskis, L. Varani and L. Reggiani, AIP Conf. Proc. 1129, 179 (2009).

[21] C. Jacoboni and P. Lugli, The Monte Carlo method for semiconductor device simulation, (Springer-Verlag, New York, (1989).

[22] J. Mateos, B. G. Vasallo, D. Pardo, T. González, J. S. Galloo, Y. Roelens, S. Bollaert and A. Cappy, Nanotechnology 14, 117 (2003).

[23] A. Íñiguez-de-la-Torre, J. Mateos, I. Íñiguez-de-la-Torre, and T. González, 8th Spanish Conference on Electron Devices, 2011. 


\section{LIST OF FIGURES}

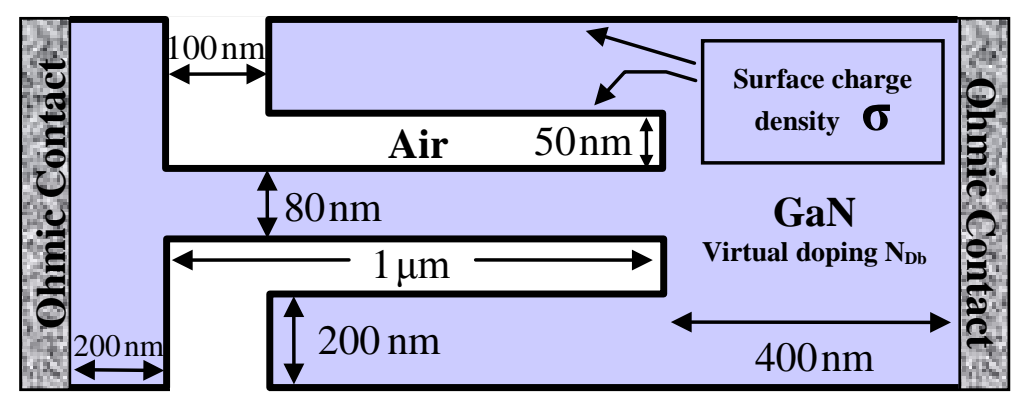

FIGURE 1. Scheme of the top-view simulations of the channel with the involved parameters, $N_{D b}$ and $\sigma$. 


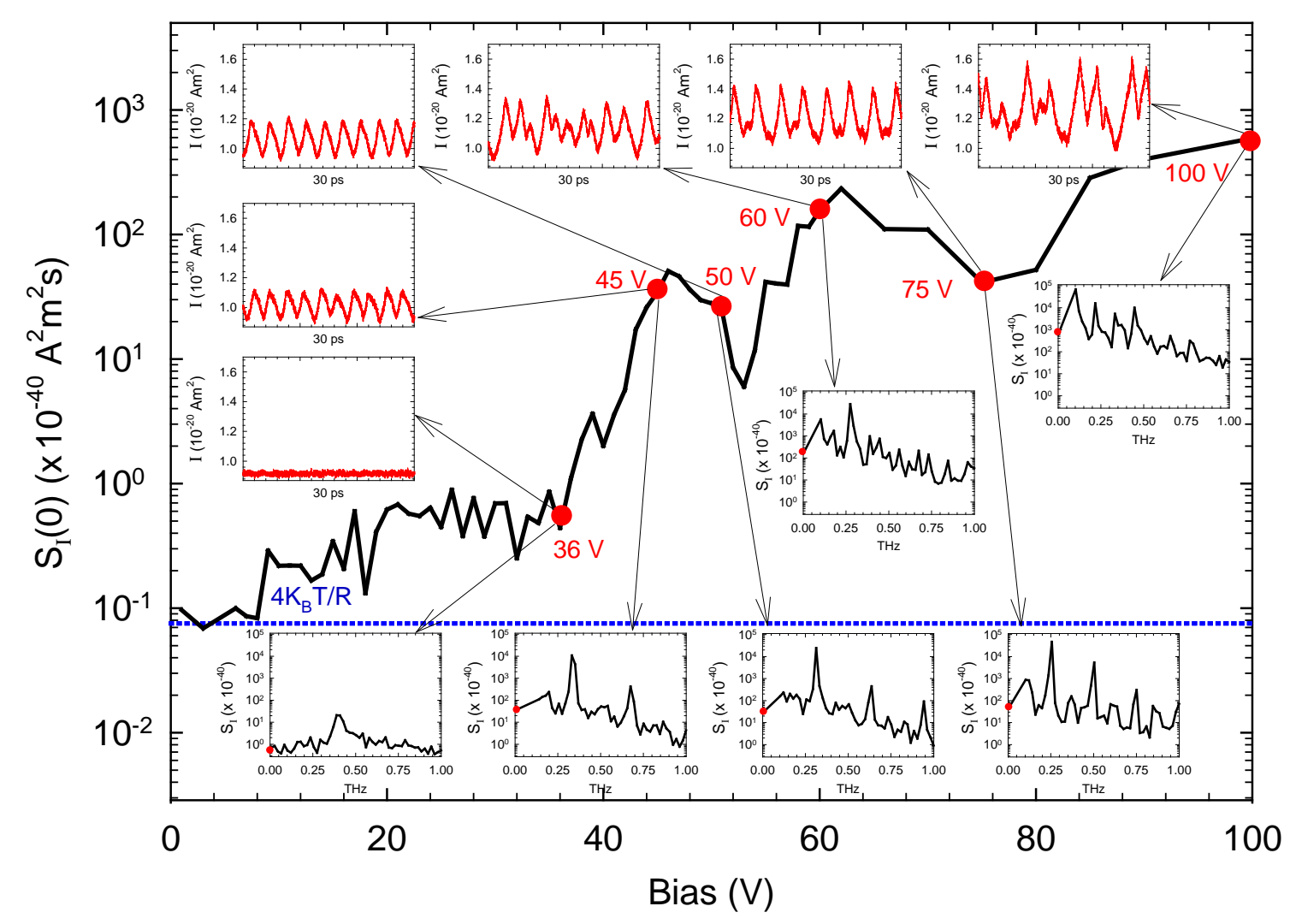

FIGURE 2. Low-frequency value of the current spectral density as a function of the bias for SSD1, where $\sigma / q=-0.25 \times 10^{12} \mathrm{~cm}^{-2}$. The insets show current sequences and spectral density as a function of frequency for several significant applied voltages. 

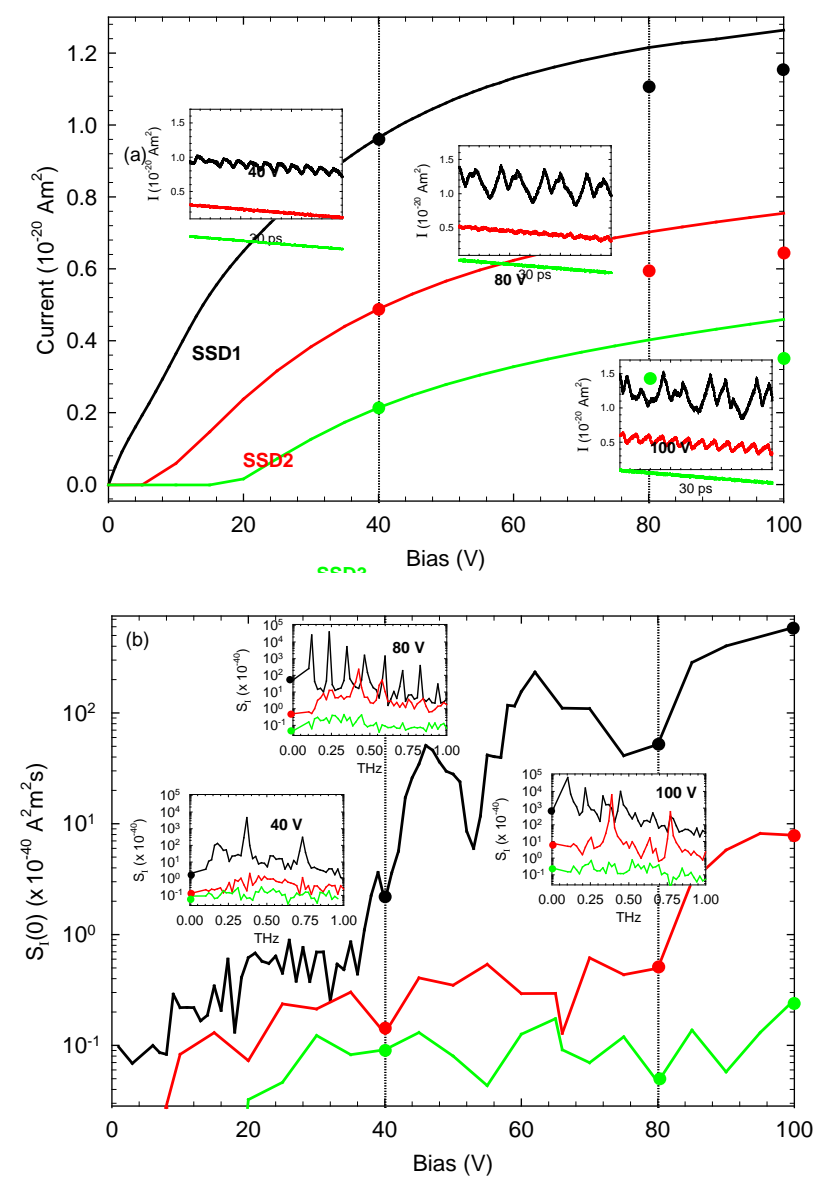

FIGURE 3. (a) $I$ - $V$ curves and (b) low-frequency value of the current spectral density for SSD1 (same as in Fig. 2) that has $\sigma / q=-0.25 \times 10^{12} \mathrm{~cm}^{-2}$, and two more diodes, SSD2 and SSD3, with $\sigma / q=-1.0 \times 10^{12}$ $\mathrm{cm}^{-2}$ and $\sigma / q=-1.5 \times 10^{12} \mathrm{~cm}^{-2}$, respectively. The insets in (a) show current sequences and noise spectra for some applied voltages and in (b) the corresponding spectra. 